\title{
Correction to: Topology Validator - Defense Against Topology Poisoning Attack in SDN
}

Abhay Kumar and Sandeep Shukla

\author{
Correction to: \\ Chapter "Topology Validator - Defense Against Topology \\ Poisoning Attack in SDN" in: X. Yuan et al. (Eds.): \\ Quality, Reliability, Security and Robustness \\ in Heterogeneous Systems, LNICST 402, \\ https://doi.org/10.1007/978-3-030-91424-0_15
}

In an older version of this paper, the "l" was missing from the last name of Sandeep

Shukla. This has been corrected. 